\title{
Effect of Tyrosine Phosphorylation of HPV31 E2 on Replication
}

\author{
Mitra Sharifi, Timra Gilson and Elliot Androphy \\ Indiana University School of Medicine
}

\section{Background}

The HPV early E2 protein is an essential regulatory protein involved in HPV replication and transcription that is encoded by all papillomaviruses. Phosphorylation of E2 at Y102 inhibits viral replication, and an additional phosphorylation site at $Y 87$ was recently identified through mass spectrometry. This project aimed to characterize E2 proteins encoding mutations at $Y 87$ that abrogate phosphorylation. In parallel, we aimed to identify the kinase responsible for E2 phosphorylation at Y87. The Androphy lab previously has published that FGFR tyrosine kinases effect HPV replication, but there may be other kinases which act upon E2. Tyk2 is a tyrosine kinase regulated by the viral protein $\mathrm{E} 6$ and highly expressed in keratinocytes. We want to determine if Tyk2 is involved in regulation of E2 through phosphorylation.

\section{Experimental Design}

We will co-express Tyk2 and E2 in 293TT cells and through immunoprecipitations determine if Tyk2 binds E2. Using an in-vitro replication assay, viral proteins E2 and E1 will be overexpressed along with Tyk2 to determine if Tyk2 effects replication. We will make HPV genomes with Y87E/Y87F mutations through site-directed mutagenesis. Expression of mutant E2 will be examined through transfection of cells and western blot analysis.

\section{Results}

E2 harboring 87 to glutamic acid (Y87E) or phenylalanine (Y87F) mutations produces soluble protein, but $87 \mathrm{E}$ inhibits replication. Tyk2 expression stabilizes E2 suggesting it binds E2.

\section{Potential impact}

It is important to understanding the mechanisms underlying a virus' replication cycle for future targeted treatments against infection. 